\title{
PERFORMANCE OF UNITARY SPACE-TIME MODULATION IN A CONTINUOUSLY CHANGING CHANNEL
}

\author{
Christian B. Peel and A. Lee Swindlehurst \\ Brigham Young University \\ Electrical and Computer Engineering Dept. \\ 459 CB, Provo, UT 84602 \\ chris.peel@ieee.org, swindle@ee.byu.edu
}

\begin{abstract}
The fading channel is a significant problem in many communications environments. In this paper, we examine the performance of unitary space-time modulation in a time-varying channel. We use a Gauss-Markov model of the continuously varying channel to characterize performance of differential and trained modulation. We find a performance ceiling at high SNR where the effect of the changing channel dominates. We show that while trained modulation provides an advantage at low SNR, above a certain SNR differential modulation gives better performance. We conclude with simulation results that support our analysis.
\end{abstract}

\section{INTRODUCTION}

Recent results from information-theoretic analysis of multiple antenna systems promise robust data transfer at high data rates [1, 2]. Recognizing that the assumption of a known channel made in these initial investigations may not always hold, Marzetta and Hochwald investigated capacity when neither the transmitter nor receiver know the channel [3], and proposed unitary signal matrices as a means of achieving capacity [4]. These codes may be viewed as a multiple-antenna extension of phase-shift-keying (PSK).

Hughes [5] and Hochwald et al. [6] apply these signals to the unknown channel by extending differential phase-shift keying (DPSK) ideas to the multiple antenna case. Tarokh also discusses differential modulation with orthogonal signals in [7]. All of these researchers assume that the channel is approximately constant for a coherence interval. Because these techniques do not require channel estimation, a potential advantage is seen over trained modulation, where a significant percentage of the coherence interval may be required for training.

The piecewise-constant model for the time-varying channel coefficients assumed in these papers is useful for several reasons. It accurately describes the way a channel might appear in a timedivision multiple access or frequency-hopping system, and its effects are simple to analyze. In other applications, however, its inability to account for the memory of the channel make it less attractive. To incorporate channel memory into our analysis, we adopt a first-order auto-regressive model for the time-variations of the channel coefficients.

In [8] we investigated a model that assumes the channel is constant for an entire symbol, but changes from symbol to symbol according to a Gauss-Markov model. This model provides a

This work was supported by the National Science Foundation under Wireless Initiative Grant CCR 99-79452. ceiling at high signal to noise ratio (SNR) beyond which increasing transmit power provides no benefit. In this paper we analyze performance of unitary space-time block codes when the channel is allowed to vary within a symbol. This model more accurately describes performance in a rapidly time-varying channel and with large numbers of transmit antennas.

\section{BACKGROUND}

We begin by describing space time block coding over the standard Rayleigh fading channel, followed by the application of a firstorder Gauss-Markov process to models of the mobile fading channel. In what follows, we let $\mathcal{C N}(0,1)$ denote a zero-mean, unitvariance, circularly symmetric complex Gaussian distribution. We call a matrix isotropically distributed (i.d.) if its elements are independent $\mathcal{C N}(0,1)$ random variables. Also, $I_{T}$ indicates the $T \times T$ identity matrix.

\subsection{Space-Time Coding in Rayleigh Fading}

Assume a Rayleigh flat-fading communications environment with $M$ transmit and $N$ receive antennas. A complex channel coefficient describes the effect of the propagation between each pair of transmit and receive antennas. These channel coefficients are assumed to be independent from element to element across the antenna array, but dependent with time. At each receive antenna, interference and other disturbances add temporally and spatially independent noise to the signal.

Consider $T$ consecutive time instants. Let $\mathbf{s}_{i}^{T}$ be the $M$ dimensional signal vector transmitted at time $i \in 0,1 \ldots, T-1$ from the antenna array, $H_{i}$ be the $M \times N$ matrix of channel coefficients seen by $\mathrm{s}_{i}^{T}$, and $W$ be a $T \times N$ matrix of i.d. additive noise. Then the $T \times N$ matrix of received data is

$$
X=\sqrt{\frac{\rho}{M}} \mathcal{S H}+W,
$$

where $\mathcal{S}$ is a $T \times T M$ block matrix containing $\mathrm{s}_{i}^{T}$ as block diagonal elements, and $\mathcal{H}$ as the $T M \times N$ block matrix obtained by stacking $H_{i}$ :

$\mathcal{S}=\left[\begin{array}{cccc}\mathbf{s}_{0}^{T} & \mathbf{0} & \cdots & \mathbf{0} \\ \mathbf{0} & \mathbf{s}_{1}^{T} & \cdots & \mathbf{0} \\ \vdots & \vdots & \ddots & \vdots \\ \mathbf{0} & \mathbf{0} & \cdots & \mathbf{s}_{T-1}^{T}\end{array}\right]$, and $\mathcal{H}=\left[\begin{array}{c}H_{0} \\ H_{1} \\ \vdots \\ H_{T-1}\end{array}\right]$ 
In the case where the channel is constant $\left(H_{i}=H\right.$, for $i \epsilon$ $0,1, \ldots, T-1)$ then $(1)$ reduces to the piecewise-constant model of [4]:

$$
X=\sqrt{\frac{\rho}{M}} S H+W,
$$

where $S$ is the $T \times M$ signal matrix having $\mathbf{s}_{i}^{T}$ as rows. We refer to $S$ as the space-time symbol transmitted in a given block of $T$ time instants. The values in this expression are normalized so that $\rho$ represents the SNR expected at each receive antenna, and does not depend on the number of transmit antennas. Modulation and performance using this model are discussed in $[3,4]$ for both known and unknown channels.

Though a piecewise-constant channel model is theoretically attractive, it is not always realistic, especially for environments with rapidly moving users. In such situations (1) is more applicable. Using the Gauss-Markov model of the fading coefficients presented next, we will show that (1) can be presented in a form similar to (3) with a diagonal matrix modifying the signal strength at each time instant. This varying signal strength will account for the effects of the channel time variations.

\subsection{Differential vs. Trained Modulation}

Space-time coding algorithms often assume that the receiver knows the channel. This knowledge is usually obtained by transmitting known symbols, which the receiver uses to estimate the channel. This estimate is then used for decoding subsequent symbols over which the channel is assumed to be constant. We refer to this as trained channel modulation.

One method of modulation with an unknown channel is differential unitary space-time modulation $[5,6]$ which assumes that the channel is constant over each pair of consecutive symbols. This scheme uses data at the current and previous time instants for encoding and decoding. The channel matrices are assumed to be equal at symbols $\tau$ and $\tau-1$ and are denoted without subscript by $H$. The current signal matrix is a unitary rotation of the previous signal: $S_{\tau}=V_{z_{\tau}} S_{\tau-1}$, where $z_{\tau} \in 0, \ldots, L-1$ indexes the unitary constellation matrix to be transmitted. Using these definitions, and working with the current received data $X_{\tau}$, the following expressions are obtained in [6]:

$$
\begin{aligned}
X_{\tau} & =\sqrt{\frac{\rho}{M}} V_{z_{\tau}} S_{\tau-1} H+W_{\tau}+(1-1) V_{z_{\tau}} W_{\tau-1} \\
& =V_{z_{\tau}} X_{\tau-1}+W_{\tau}-V_{z_{\tau}} W_{\tau-1} \\
& =V_{z_{\tau}} X_{\tau-1}+\sqrt{2} \hat{W}_{\tau} .
\end{aligned}
$$

In (4), $V_{z_{\tau}} W_{\tau-1}$ is added and subtracted from (3), resulting in (5) which does not explicitly depend on $H$. Finally, because the noise matrices are statistically invariant to multiplication by unitary matrices, (6) is obtained, which is called the "fundamental differential receiver equation" in [6].

Because the effective channel $\left(X_{\tau-1}\right)$ has signal strength $\rho$, the system has an effective SNR of $\rho / 2$. This factor of 2 is the multiple-antenna generalization of the well-known $3 \mathrm{db}$ loss in performance when using DPSK versus coherent PSK.

\subsection{A Gauss-Markov Fading Channel Model}

In Section 3, we analyze the performance of unitary space-time modulation under the assumption that the channel matrix $H_{t}$ occurs $r$ samples after a reference (or estimated) channel $H_{\text {ref }}=$
$H_{t-r}$. We assume that $H_{t}$ varies from that reference channel according to the following first-order auto-regressive (AR) model ${ }^{1}$ :

$$
H_{t}=\sqrt{\alpha_{r}} H_{\text {ref }}+\sqrt{1-\alpha_{r}} E_{t},
$$

where $H_{0}$ and $E_{t}$ are i.d., $E_{t}$ is independent from symbol to symbol, $\alpha_{r} \in \mathbb{R}$ and $0 \leq \alpha_{r} \leq 1$. Under this model, $H_{t}$ is i.d., and thus (7) is a first-order Gauss-Markov process. With differential coding $r \approx T$, an d demodulation is based on the previous symbol (of length $T$ ), while typically for trained modulation $r \approx K T$, with demodulation based on a channel estimate obtained $K>1$ symbols in the past. In general we will allow different values for $r$ (and thus for $\alpha_{r}$ ) for each time instant within a symbol. Note that $\alpha_{r}=1$ means a time-invariant channel, and $\alpha_{r}=0$ indicates a completely random time-varying channel.

The AR parameter $\alpha_{r}$ can be chosen, for example, to match the second order statistics of models based on the mechanisms of physical propagation. Let $r_{h h}(t)$ denote the autocorrelation function of an element of $H$. We focus on Jakes' model of the land mobile fading channel [9], where $r_{h h}(t)=J_{0}(2 \pi f t)$. In this equation, $J_{0}(\cdot)$ is the zeroth-order Bessel function of the first kind, $f=f_{d} T_{s}, f_{d}$ is the maximum Doppler frequency in the fading environment, and $T_{s}$ is the sampling period. Solving the Yule-Walker equations for $\alpha_{r}$ in the first-order AR process (7) gives

$$
\alpha_{r}=\left[\frac{r_{h h}(r)}{r_{h h}(0)}\right]^{2}=J_{0}(2 \pi r f)^{2}
$$

which provides a reasonable choice for $\alpha_{r}$. This AR model is an appropriate approximation to Jakes' model for realistic (small) values of $f$ when using the maximum-likelihood decoders of [4] which depend on a single reference channel. This fact is borne out by the simulation results of Section 4 , where excellent agreement is obtained with data generated according to Jakes' model, but analyzed with the AR model using (8).

\section{PERFORMANCE IN THE CONTINUOUSLY CHANGING CHANNEL}

In this section, we analyze unitary space-time coding using the channel model (1) which allows a different channel matrix at each time instant. Because the channel at the end of a symbol has changed the most, we expect the effective signal power for the last signal elements transmitted to be lower than for the first. Our analysis below supports this intuition.

\subsection{Trained Modulation}

We initially look at performance $R$ time samples after the reference channel is obtained. By letting $R=K T$, this models the performance of trained modulation $K$ symbols (of length $T$ ) after training. We assume that the reference channel is error-free and thus do not account for estimation error in our model.

Theorem 1. Given the data model of (1), assume that the channel at each time instant varies from a reference channel $H_{\text {ref }}$ according to (7). The effect of the time-varying channel $R$ time samples after the reference is that of a time-varying effective SNR and is described by the following equation

$$
X=\sqrt{\frac{\rho}{M}} P_{R} S H_{\mathrm{ref}}+\bar{W},
$$

\footnotetext{
${ }^{1}$ Higher order models may be appropriate for analysis of more complex decoders than those using a single reference channel (which are assumed here).
} 
where $P_{R}$ is the diagonal matrix formed from $p_{0}, p_{1}, \cdots, p_{T-1}$, and

$$
p_{i}=\sqrt{\frac{\alpha_{R+i}}{1+\left(1-\alpha_{R+i}\right) \rho \frac{T}{M}}} \leq 1 .
$$

If the reference channel is from more than a symbol in the past $(R>T)$ then $p_{i}<p_{i-1}$

Proof. Starting with (2), we substitute in for each channel matrix to obtain

$$
\dot{\mathcal{H}}=\left[\begin{array}{c}
\sqrt{\alpha_{R}} H_{\mathrm{ref}}+\sqrt{1-\alpha_{R}} E_{0} \\
\sqrt{\alpha_{R+1}} H_{\mathrm{ref}}+\sqrt{1-\alpha_{R+1}} E_{1} \\
\vdots \\
\sqrt{\alpha_{R+T-1}} H_{\mathrm{ref}}+\sqrt{1-\alpha_{R+T-1}} E_{T-1}
\end{array}\right] .
$$

We first define the block matrices

$$
\tilde{\mathcal{H}}=\left[\begin{array}{c}
H_{\text {ref }} \\
\vdots \\
H_{\text {ref }}
\end{array}\right] \quad \mathcal{E}=\left[\begin{array}{c}
E_{0} \\
\vdots \\
E_{T-1}
\end{array}\right],
$$

and the diagonal matrix

$$
D_{R}=\operatorname{diag}\left(\alpha_{R}, \alpha_{R+1}, \ldots, \alpha_{R+T-1}\right) .
$$

Thus $D_{R}$ and $\left(I_{T}-D_{R}\right)$ contain the power in the reference channel and noise portions of $\mathcal{H}$, which we may now write in terms of these matrices:

$$
\mathcal{H}=\left(D_{R}^{\frac{1}{2}} \otimes I_{M}\right) \tilde{\mathcal{H}}+\left[\left(I_{T}-D_{R}\right)^{\frac{1}{2}} \otimes I_{M}\right] \mathcal{E} .
$$

Substituting (14) into (1), we obtain

$$
\begin{aligned}
X= & \sqrt{\frac{\rho}{M}} \mathcal{S}\left(D_{R}^{\frac{1}{2}} \otimes I_{M}\right) \tilde{\mathcal{H}}+ \\
& \sqrt{\frac{\rho}{M}} \mathcal{S}\left[\left(I_{T}-D_{R}\right)^{\frac{1}{2}} \otimes I_{M}\right] \mathcal{E}+W .
\end{aligned}
$$

Because

$$
\mathcal{S}\left(D_{R}^{\frac{1}{2}} \otimes I_{M}\right) \overline{\mathcal{H}}=D_{R}^{\frac{1}{2}} S H_{\text {ref }},
$$

also because $S$ is $\sqrt{T}$ times a unitary matrix [4], then

$$
\sqrt{\frac{\rho}{M}} \mathcal{S}\left[\left(I_{T}-D_{R}\right)^{\frac{1}{2}} \otimes I_{M}\right] \mathcal{E}=\sqrt{\rho \frac{T}{M}}\left(I_{T}-D_{R}\right)^{\frac{1}{2}} \hat{E}
$$

where $\hat{E}$ is i. d., and we may write

$$
X=\sqrt{\frac{\rho}{M}} D_{R}^{\frac{1}{2}} S H_{\mathrm{ref}}+\sqrt{\rho \frac{T}{M}}\left(I_{T}-D_{R}\right)^{\frac{1}{2}} \hat{E}+W .
$$

Adding the variances of the two noise matrices and combining them, and then normalizing to obtain unit variance we obtain

$$
X=\sqrt{\frac{\rho}{M}}\left(D_{R}^{\frac{1}{2}}\left[I+\left(I_{T}-D_{R}\right) \rho \frac{T}{M}\right]^{-\frac{1}{2}}\right) S H_{\mathrm{ref}}+\tilde{W} .
$$

Recognizing that the terms in parentheses are an alternate form for $P_{R}$, we have obtained (9).

The product $\rho p_{i}^{2}$ may be viewed as the effective SNR (ESNR) seen by $\mathbf{s}_{i}^{T}$. We see that as time progresses within a symbol matrix, the ESNR also decreases (assuming $R>T$ ); the more time that has elapsed since the last training estimate, the lower the effective SNR at each sample. Because $P_{R}$ modulates the signal strength with time, we will use $P_{R} S$ as the transmitted signal in place of $S$ in the probability expressions from [4] when comparing simulation with this analysis in Section 4.

It is instructive to look at what happens to $P_{R}$ for limiting values of $\alpha_{i}$. Note that $\lim _{\alpha_{i} \rightarrow 1} P_{R}=I_{M}$, which merely indicates that for a constant channel, the effect of $P_{R}$ disappears, and (3) applies. In the other direction, $\lim _{\alpha_{i} \rightarrow 0} P_{R}=0$, indicating that as channel speed increases, the effective SNR will drop to zero. As expected, we also see that $\lim _{\rho \rightarrow 0} P_{R}=0$. As $\rho$ goes to infinity we find that the ESNR at each instant ceases to depend on $\rho$, and depends solely on the parameters of the changing channel:

$$
\lim _{\rho \rightarrow \infty} \sqrt{\rho} P_{R}=\operatorname{diag}\left(\sqrt{\frac{\alpha_{R}}{1-\alpha_{R}}}, \cdots, \sqrt{\frac{\alpha_{R+T-1}}{1-\alpha_{R+T-1}}}\right) .
$$

\subsection{Differential Modulation}

We now use the continuously-varying model (1) and the time varying channel (7) to analyze differential unitary modulation. Recall that for differential modulation, the previous received data is the effective channel, and thus it makes sense to use the channel seen by the previous symbol as the reference. This doesn't solve our problem, however, because there is a different channel seen by the previous symbol at each of the $T$ time instants. We desire to place the reference channel in the position which is closest to the other channels seen by the previous symbol. We choose to use as reference the channel that is temporally in the center of the previous symbol; the first time sample in the current symbol will thus be $\frac{T+1}{2}$ time samples away from the reference.

Theorem 2. Given the data model of (1), assume that the channel at each time instant varies according to (7). The effect of the time-varying channel on differential modulation is that of a timevarying and decreasing effective SNR, and is described by the following equation

$$
X_{t}=P_{\mathrm{Dif}} V_{z_{l}} X_{t-1}+\vec{W}_{t},
$$

where $P_{\mathrm{Dif}}$ is the signal-dependent matrix:

$$
\begin{aligned}
P_{\text {Dif }}= & D_{-R_{m}}^{\frac{1}{2}}\left[I_{T}+D_{-R_{m}} D_{R_{p}}^{-1}+\left(I_{T}+\right.\right. \\
& \left.\left.D_{-R_{m}} D_{R_{p}}^{-1}-D_{-R_{m}}-V_{z_{\tau}} D_{-R_{m}} V_{z_{\tau}}^{*}\right) \rho \frac{T}{M}\right]^{-\frac{1}{2}},
\end{aligned}
$$

where $R_{m}=\frac{T-1}{2}$, and $R_{p}=\frac{T+1}{2}$, and $D_{R}$ is as defined in (13). Proof. Using Theorem 1 we may write the current and previous received data as

$$
\begin{aligned}
\mathcal{X}_{\tau-1} & =\sqrt{\frac{\rho}{M}} S_{\tau-1} H_{\mathrm{ref}}+P_{-R_{m}}^{-1} \hat{W}_{\tau} \\
\mathcal{X}_{\tau} & =\sqrt{\frac{\rho}{M}} V_{z_{\tau}} S_{\tau-1} H_{\mathrm{ref}}+P_{R_{p}}^{-1} \hat{W}_{\tau} .
\end{aligned}
$$

Adding and subtracting the noise term from (23) rotated by $V_{z_{\tau}}$ to (24) we obtain

$$
\begin{aligned}
\mathcal{X}_{\tau}= & V_{z_{\tau}}\left(\sqrt{\frac{\rho}{M}} S_{\tau-1} H_{\mathrm{ref}}+P_{-R_{m}}^{-1} \hat{W}_{\tau}\right) \\
& +P_{R_{p}}^{-1} \hat{W}_{\tau}-V_{z \tau} P_{-R_{m}}^{-1} \hat{W}_{\tau} .
\end{aligned}
$$

The terms in parentheses combine to form $X_{\tau-1}$, and we may combine the two noise terms as follows:

$$
\mathcal{X}_{\tau}=V_{z_{\tau}} X_{\tau-1}+\left[P_{R_{p}}^{-2}+V_{z \tau} P_{-R_{m}}^{-2} V_{z \tau}^{*}\right]^{\frac{1}{2}} \hat{W}_{\tau} .
$$

Normalizing to obtain noise with unit variance, we obtain an equation for $X_{\tau}$ which has the same SNR as $\mathcal{X}_{\tau}$ :

$$
X_{\tau}=\left[P_{R_{p}}^{-2}+V_{z \tau} P_{-R_{m}}^{-2} V_{z \tau}^{*}\right]^{-\frac{1}{2}} V_{z_{\tau}} X_{\tau-1}+\tilde{W}_{\tau} .
$$

With the alternate representation of $P_{\mathrm{Dif}}$ as

$$
P_{\mathrm{Dif}}=\left[P_{R_{p}}^{-2}-V_{z \tau} P_{-R_{m}}^{-2} V_{z \tau}^{*}\right]^{-\frac{1}{2}}
$$




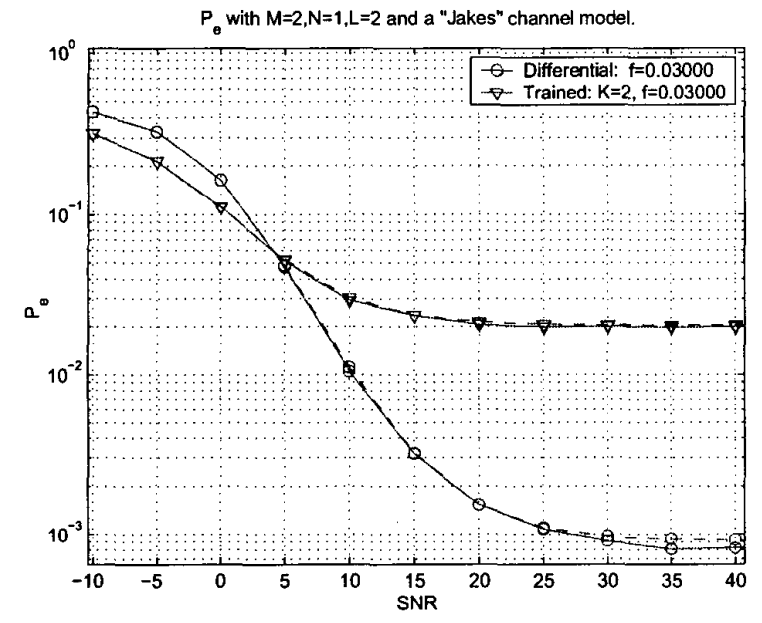

Fig. 1. Comparing the two channel models.

a bit of matrix algebra is sufficient to show that (27) is (21).

This result suggests a simple technique for determining the performance of differential unitary space-time coding techniques. Because the matrix $P_{\text {Dif }}$ modulates the signal strength with time, we replace the signal matrix $V_{z_{\tau}}$ with $P_{\mathrm{Dif}} V_{z_{\tau}}$ in probability of error expressions [4] when comparing simulation with our analysis in Section 4.

Note that if the signals are diagonal, then $P_{\text {Dif }}$ loses its dependence on the signal, and we obtain

$$
\begin{aligned}
P_{\mathrm{Dif}}= & D_{-R_{m}}^{\frac{1}{2}}\left[I_{T}+D_{-R_{m}} D_{R_{p}}^{-1}+\left(I_{T}+\right.\right. \\
& \left.\left.D_{R_{p}}^{-1} D_{-R_{m}}-2 D_{-R_{m}}\right) \rho \frac{T}{M}\right]^{-\frac{1}{2}}
\end{aligned}
$$

Again, it is useful to look at limiting values for these ESNR matrices. Note that $\lim _{\alpha_{i} \rightarrow 1} P_{\mathrm{Dif}}=\frac{1}{2} I_{M}$ which merely indicates that for a constant channel, the effect of $P_{\text {Dif }}$ disappears, and (6) applies. The interesting case is to see what happens as $\rho$ goes to infinity, or when errors due to noise become less important and errors due to the changing channel become dominant:

$$
\begin{aligned}
\lim _{\rho \rightarrow \infty} \sqrt{\rho} P_{\text {Dif }}= & D_{-R_{m}}^{\frac{1}{2}}\left[\left(I_{T}+D_{R_{p}}^{-1} D_{-R_{m}}-\right.\right. \\
& \left.\left.D_{-R_{m}}-V_{z_{\tau}} D_{-R_{m}} V_{z_{\tau}}^{*}\right) \frac{T}{M}\right]^{-\frac{1}{2}} .
\end{aligned}
$$

As expected, we find at high SNR that performance is limited by the time-varying channel and not by the noise.

\section{RESULTS}

We have presented analytic results quantifying performance for a continuously varying fading channel with memory. We now present simulation results that support our analysis. We use $L=2$ signals in our constellations which consist simply of the identity and negative identity matrices. Though our results hold true for all unitary signal matrices, we use these constellations because of the resulting simple analytic expressions for probability of error.
We present results for $M=2$ transmit antennas, $N=1$ receive antennas, training interval $K=2$, and a fading parameter of $f=0.03$. This corresponds to the Doppler shift obtained at sixty miles per hour with a carrier to bandwidth ratio of $333,333 / 1$; this illustrates the utility of our analysis in a fading environment. Use of the ESNR parameter with $\alpha_{K T}$ from (8) in place of the true SNR in the probability of error expressions in [4] as discussed in [8] gives the analytic results for trained and differential modulation shown with the dotted lines in Figure 1. The dashed lines show analytic results with the continuously changing model presented in Section 3. These were obtained by replacing the signal matrices in the pairwise probability of error expressions from [4] with the product of the ESNR matrices and the signal matrices. The solid lines give the results of simulations with channel coefficients that obey Jakes' model. We generated channel coefficients with Jakes' fading simulator, and simulated six million symbols at each SNR value shown to calculate the probability of error results. Though there is a three $\mathrm{db}$ advantage for trained modulation apparent at low SNR, at high SNR differential modulation provides a significant advantage. Our analytic and simulation results ${ }^{2} \mathrm{ma}$ tch very well for both differential and trained modulation.

\section{REFERENCES}

[1] I. E. Telatar, "Capacity of multi-antenna gaussian channels," European Transactions on Telecommunications, vol. 10, no. 6, pp. 585-595, Nov/Dec 1999.

[2] G. J. Foschini and M. J. Gans, "On limits of wireless communications in a fading environment when using multiple antennas," Wireless Personal Communications, vol. 6, pp. 311-335, 1998.

[3] T. L. Marzetta and B. M. Hochwald, "Capacity of a mobile muliple-antenna communication link in Rayleigh flat fading," IEEE Transactions on Information Theory, vol. 45, no. 1, pp. 139-157, May 1999.

[4] B. M. Hochwald and T. L. Marzetta, "Unitary space-time modulation for multiple-antenna communications in rayleigh flat fading," IEEE Transactions on Information Theory, vol. 46, no. 2, pp. 543-564, March 2000.

[5] B. L. Hughes, "Differential space-time modulation," in Proceedings of the IEEE Wireless Communications and and Networking Conference, New Orleans, September 1999, vol. 1, pp. 145-149.

[6] B. M. Hochwald and W. Sweldens, "Differential unitary space-time modulation," To appear in IEEE Trans. Comm., March 1999.

[7] V. Tarokh and H. Jafarkani, "A differential detection scheme for transmit diversity," IEEE Journal on Selected Areas in Communications, vol. 18, no. 3, pp. 1169-1174, July 2000.

[8] C. B. Peel and A. L. Swindlehurst, "Peformance of unitary space-time modulation in rayleigh fading," in Proc. International Conference on Communications ICC 2001, Helsinki, Finland, June 2001.

[9] W. C. Jakes, Microwave Mobile Communications, IEEE Press, 1993.

${ }^{2}$ Matlab software used in generate the results in this paper is available at http://www.ee.byu.edu/ee/wireless/or by contacting the first author 